\title{
Time-domain measurement of optical activity by an ultrastable common-path interferometer
}

\author{
Fabrizio Preda, ${ }^{1}$ (1) Antonio Perri, ${ }^{1}$ (i) Julien Réhault, ${ }^{2}$ (i) Biplab Dutta, ${ }^{3}$ Jan Helbing, ${ }^{3}$ (i) \\ Giulio Cerullo, ${ }^{1}$ (i) and Dario Polli ${ }^{1,4, *}$ (1) \\ ${ }^{1}$ IFN-CNR, Dipartimento di Fisica, Politecnico di Milano, Piazza Leonardo da Vinci 32, I-20133 Milano, Italy \\ ${ }^{2}$ Institute of Applied Physics, University of Bern, Sidlerstrasse 5, 3012 Bern, Switzerland \\ ${ }^{3}$ University of Zurich, Department of Chemistry, Winterthurerstrasse 190, 8057 Zurich, Switzerland \\ ${ }^{4}$ Center for Nano Science and Technology@PoliMi, Istituto Italiano di Tecnologia, Via Giovanni Pascoli 70/3, 20133 Milano, Italy \\ *Corresponding author: dario.polli@polimi.it
}

Received 5 March 2018; accepted 15 March 2018; posted 21 March 2018 (Doc. ID 325172); published 12 April 2018

\begin{abstract}
We introduce a novel configuration for the broadband measurement of the optical activity of molecules, combining time-domain detection with heterodyne amplification. A birefringent common-path polarization-division interferometer creates two phase-locked replicas of the input light with orthogonal polarization. The more intense replica interacts with the sample, producing a chiral free-induction decay field, which interferes with the other replica, acting as a time-delayed phase-coherent local oscillator. By recording the delay-dependent interferogram, we obtain by a Fourier transform both the circular dichroism and circular birefringence spectra. Our compact, low-cost setup accepts ultrashort light pulses, making it suitable for measurement of transient optical activity. (C)2018 Optical Society of America
\end{abstract}

OCIS codes: (260.2130) Ellipsometry and polarimetry; (300.6310) Spectroscopy, heterodyne; (320.7100) Ultrafast measurements.

https://doi.org/10.1364/OL.43.001882

Provided under the terms of the OSA Open Access Publishing Agreement

The chirality of molecules is of paramount importance in biology and chemistry, as it has a strong influence on their biochemical functions; for this reason, its measurement is required for many applications in the chemical, pharmaceutic, and food industries. Interaction of chiral molecules with light gives rise to optical activity $(\mathrm{OA})[1,2]$, which manifests itself in circular dichroism $(\mathrm{CD})$ and circular birefringence $(\mathrm{CB})$, i.e., difference in absorbance and refraction, respectively, between left (LCP) and right (RCP) circularly polarized light. OA also arises from the chiral arrangement of chromophores in biopolymers, such as proteins and nucleic acids. Ultraviolet (UV) CD spectroscopy can thus be used to determine the secondary structure content of proteins as well as study the proteins' folding processes $[3,4]$. When a linearly polarized light field impinges on an achiral medium, it drives a time-varying material polarization that in turn radiates a light field, the achiral-free induction decay (AFID); interference of the AFID with the input field gives rise to linear absorption/refraction. A chiral medium, conversely, radiates a small light field with perpendicular polarization, known as chiral FID (CFID), which changes the polarization state of the output light, turning it from linear to elliptical $[5,6]$. As the CFID field is very weak, $C D$ and $C B$ are orders of magnitude smaller than the corresponding achiral absorption/refraction, making their experimental measurement challenging. Standard CD spectrometers, also known as spectropolarimeters (SP), extract the differential absorption of the RCP and LCP light via fast polarization modulation and synchronous detection. In the visible/UV range, they work in the frequency domain: a photoelastic modulator (PEM) switches the polarization of a monochromatized light beam between RCP and LCP at tens of kilohertz frequency, and a lock-in amplifier measures the tiny absorbance difference. By scanning the excitation wavelength, it is possible to record a $\mathrm{CD}$ spectrum with very high sensitivity. In the mid-IR, vibrational CD (VCD) is typically measured in the time domain by Fourier-transform (FT) IR spectrometers, in which the output polarization of an amplitude division interferometer (such as a Michelson) is modulated by a PEM. These approaches require complex, bulky and expensive instruments.

Alternative configurations for measuring $\mathrm{CD}$ use a polarization-division interferometer (PDI), which is a modified amplitude-division interferometer in which the light in the two arms is linearly polarized in perpendicular directions [7]. Ragunathan et al. [8] and Polavarapu et al. [9] used a Michelson interferometer modified with polarizing beamsplitters to record VCD spectra in the time domain. As the optical path length difference between the arms is varied, the polarization of the interferometer output quickly changes between LCP and RCP. Only in the presence of a chiral sample can an interferogram (IG) be recorded, and the $\mathrm{CD}$ spectrum is retrieved by a FT. Rhee et al. [10,11] used a free-space Mach-Zehnder interferometer and employed spectral interferometry in the frequency domain [12]. The interferometer generated an intense vertically polarized replica $(\mathrm{V})$ and a weaker horizontal one $(\mathrm{H})$. The interferometer delay $\tau$ was fixed and the light transmitted by the sample was dispersed on a spectrometer. The $\mathrm{V}$ replica generates a horizontally polarized CFID field in the chiral sample, which is then 
selected by an $\mathrm{H}$ analyzer. The $\mathrm{H}$ replica thus acts as a local oscillator (LO), which interferes with the CFID. This yields, via a Kramers-Kronig transform, the complex chiral signal. A second measurement with analyzer aligned along the $\mathrm{V}$ direction produces an achiral reference signal, required to extract the CD and CB spectra [13]. The main limitation of interferometric approaches is the required high path-length stability between the two arms of the interferometer, since any vibration by a fraction of wavelength would spoil the measurement. This stability is more easily achieved for VCD, which works at long mid-IR wavelengths $(\lambda>3 \mu \mathrm{m})$; at shorter wavelengths, active stabilization of the interferometer or simultaneous single-shot detection of the spectra using a dual-channel spectrometer $[14,15]$ is required.

Here we introduce a novel and simple time-domain approach to electronic OA measurements in the visible/near-IR. Our method uses an innovative PDI based on an intrinsically phase-stable birefringent common-path interferometer (CPI). It generates two phase-locked perpendicularly polarized replicas of the broadband input light without active stabilization or delay tracking. The weaker replica acts as LO, phase-locked to the CFID field generated by the other replica. By scanning the delay between replicas and recording the IG between LO and CFID with a single detector, one can simultaneously obtain with an FT both the CD and CB spectra. We demonstrate operation with both spatially incoherent and coherent light, illustrating the potential of our compact, robust, and low-cost SP. Importantly, our CPI accepts broadband ultrashort light pulses, paving the way to ultrafast detection of OA.

Figure 1 shows the conceptual scheme of a time-domain SP employing a PDI device. A first polarizer $\left(P_{1}\right)$ creates a field linearly polarized at an angle $\gamma$ with respect to the $\mathrm{V}$ direction. The PDI splits this field into two replicas with $\mathrm{V}$ and $\mathrm{H}$ polarization and delay $\tau$. As OA is a linear optical property, we can apply the superposition principle and decompose the broadband illumination into monochromatic waves with frequency $\omega$ and amplitude $E_{0}(\omega)$. For each wave the Jones vector [16] describing the electric field after the PDI is, as follows:

$$
E_{\text {in }}(\omega, \tau)=E_{0}(\omega)\left(\sin \gamma \cos \gamma \cdot e^{i \omega \tau}\right)^{T} .
$$

For an isotropic chiral sample with absorbance $\alpha=$ $\left(\alpha_{\mathrm{LCP}}+\alpha_{\mathrm{RCP}}\right) / 2$, mean refractive index $n=\left(n_{\mathrm{LCP}}+\right.$ $\left.n_{\mathrm{RCP}}\right) / 2$, and thickness $L$, the Jones matrix can be expressed, in the limit of weak CD and CB, as [17]

$$
M_{\mathrm{chi}} \approx 10^{-\alpha / 2} e^{-i \rho}\left(\begin{array}{cc}
1 & \mathrm{CB}-i \mathrm{CD} \\
-\mathrm{CB}+i \mathrm{CD} & 1
\end{array}\right),
$$

where $\mathrm{CB}=\left(n_{\mathrm{LCP}}-n_{\mathrm{RCP}}\right) L \omega /(2 c)$ and $\mathrm{CD}=\left(\alpha_{\mathrm{LCP}}-\alpha_{\mathrm{RCP}}\right)$ $\ln (10) / 4$ are the optical rotation and ellipticity in radians, $\rho=n L \omega / c$, and $c$ is the speed of light in vacuum. The electric

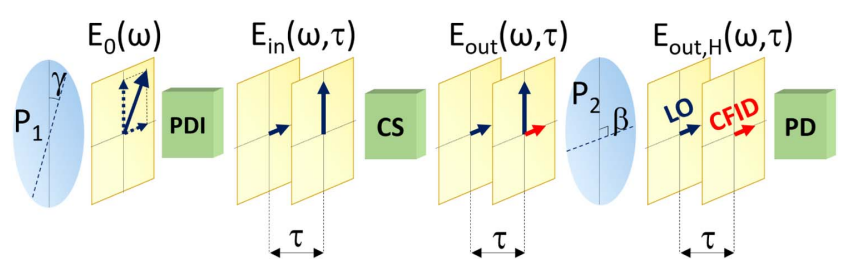

Fig. 1. Scheme of the time-domain SP. $P_{1}, P_{2}$, polarizers; PDI, polarization-division interferometer; $\mathrm{CS}$, chiral sample; $\mathrm{LO}$, local oscillator; CFID, chiral free-induction decay signal; PD, photodiode. field transmitted by the chiral sample is $E_{\text {out }}(\omega)=$ $M_{\text {chi }} E_{\text {in }}(\omega)$. After the sample, polarizer $P_{2}$ oriented along the $\mathrm{H}$ direction $\left(\beta=90^{\circ}\right.$ in Fig. 1) selects the horizontal component of $E_{\text {out }}$, as follows:

$$
\begin{aligned}
E_{\text {out }, \mathrm{H}}(\omega, \tau)= & E_{0}(\omega) 10^{-\alpha / 2} e^{-i \rho} \\
& \cdot\left[\sin \gamma+(\mathrm{CB}-i \mathrm{CD}) \cdot \cos \gamma \cdot e^{i \omega \tau}\right] .
\end{aligned}
$$

It consists of the superposition of the CFID field with the $\mathrm{H}$ component of $E_{\text {in }}$ transmitted by the sample, which acts as a LO. By measuring with a single detector the intensity transmitted by polarizer $P_{2}$ as a function of the delay $\tau$, and by integrating over all the monochromatic components, we obtain the chiral IG, as follows:

$$
\begin{aligned}
I_{\mathrm{chi}}(\tau)= & \int \mathrm{d} \omega\left|E_{\mathrm{out}, \mathrm{H}}(\omega, \tau)\right|^{2} \\
\cong & \int \mathrm{d} \omega\left|E_{0}(\omega)\right|^{2} 10^{-\alpha(\omega)} \\
& \cdot\left\{\sin ^{2} \gamma+2 \sin \gamma \cos \gamma\right. \\
& \cdot[\mathrm{CB}(\omega) \cos (\omega \tau)+\mathrm{CD}(\omega) \sin (\omega \tau)]\} .
\end{aligned}
$$

This expression contains the intensity of the LO and the mixing term between the LO and the CFID fields (selfheterodyne amplification), while we neglect the much weaker intensity of the CFID field. By taking the imaginary and the real parts of the FT of the chiral IG $I_{\text {chi }}(\tau)$, one can compute the $\mathrm{CD}$ and $\mathrm{CB}$ spectra, respectively. To obtain the absolute values of the signals, we perform a calibration measurement with the second polarizer tilted by an angle $\beta=45^{\circ}$. In this case, the two replicas generated by the PDI interfere at the detector, and the much weaker CFID can be neglected, yielding the calibration IG, as follows:

$$
I_{\mathrm{cal}}(\tau)=\int \mathrm{d} \omega\left|E_{0}(\omega)\right|^{2} 10^{-\alpha(\omega)} \cdot \frac{1}{2}\{1+\sin (2 \gamma) \cdot \cos (\omega \tau)\}
$$

The ratio of the FTs of chiral and calibration IGs gives

$$
\begin{aligned}
& \mathrm{CD}(\omega)=-1 / 2 \operatorname{Im}\left\{\operatorname{FT}\left[I_{\text {chi }}(\tau)\right] / \operatorname{FT}\left[I_{\text {cal }}(\tau)\right]\right\} \\
& \mathrm{CB}(\omega)=1 / 2 \operatorname{Re}\left\{\operatorname{FT}\left[I_{\text {chi }}(\tau)\right] / \operatorname{FT}\left[I_{\text {cal }}(\tau)\right]\right\} .
\end{aligned}
$$

These equations are very similar to those extracted from spectral interferometry by Rhee $e t a l$. [10]. The main difference is that our time-domain method directly computes the complex OA spectrum from the FTs of the chiral and calibration temporal IGs, while the frequency-domain method measures spectral IGs as real signals. Therefore, an inverse FT to the time domain, temporal filtering, and FT back to the frequency domain are required to obtain complex OA spectra.

Equation (4) shows that the chiral IG $I_{\text {chi }}(\tau)$ sits on an offset proportional to $\sin ^{2}(\gamma)$, while the amplitude of the fringe pattern is proportional to $\sin (2 \gamma)$, so that the modulation depth (defined as the amplitude of the fringes divided by the offset) for the CD and CB signals is proportional to $\cot \gamma$. Therefore, small values of $\gamma$ result in higher contrast of the fringes but reduce the amplitude of the fringe pattern, because the lower amplitude of the LO decreases the self-heterodyne amplification. In practice, the optimal choice of the polarizer angle $\gamma$ depends on the amount of light available, the dynamic range of the detector, and the quality of the polarizers. Typical values 
of $\gamma$ range between $0.5^{\circ}$ and $5^{\circ}$, thus achieving an amplification of the modulation depth by $1-2$ orders of magnitude with respect to the symmetric case $\left(\gamma=45^{\circ}\right)$ and increasing the signalto-noise ratio considerably. The largest amplification is possible with intense light sources like lasers, and this measurement principle has been used previously to significantly enhance $\mathrm{CD}$ (and $\mathrm{CB}$ ) signals in frequency-domain measurements with wavelength selection both before $[5,18]$ and after $[19,20]$ the sample. On the other hand, if only CD signals are measured, the second polarizer $\left(P_{2}\right.$ in Fig. 1) may be omitted in full analogy to the VCD setup in Ref. [9] but with the much higher delay stability and reproducibility that allows us to extend the method to the UV-visible range. In this case, the $\mathrm{H}$ and $\mathrm{V}$ components of PDI output should be of equal strength $\left(\gamma=45^{\circ}\right)$, and the modulation depth of the chiral IG cannot be amplified.

Figure 2 shows the experimental setup of our time-domain $\mathrm{SP}$, in which the PDI is a birefringent CPI. It starts with a broadband light source, which can be either coherent or incoherent. For coherent excitation, we used the near-infrared portion of the supercontinuum (SC) generated by a fiber laser, with $1-3 \mathrm{~mW} / \mathrm{nm}$ power density. As incoherent light source, we employed a halogen lamp at $2500 \mathrm{~K}$ emitting 310 lumens. The broadband illumination is sent to a first polarizer $P_{1}$ (extinction ratio $>10^{5}$ ), with transmission axis tilted from the vertical direction by a small angle $\gamma$. After the polarizer, the light passes the birefringent CPI, which is a simplified version of the Translating-Wedge-based Identical pulses eNcoding System (TWINS) [21,22]. TWINS is composed of two blocks of a birefringent material, A and B, with optical axes (see blue arrow and dots in Fig. 2) rotated by $90^{\circ}$ with respect to each other and perpendicular to the propagation direction of the beam. Block $A$ is a parallel-face plate with $V$ optical axis that introduces a fixed delay between the $\mathrm{V}$ and $\mathrm{H}$ replicas. Block $\mathrm{B}$ is shaped in the form of two identical wedges, with $\mathrm{H}$ optical axis, introducing a delay of opposite sign. One of the wedges of block B is mounted on a precise motorized translation stage, allowing a change in its insertion and thus varying the thickness of block B. To minimize lateral displacement of the beam, the direction of motion is not perpendicular to the light beam but inclined by the apex angle of the wedge (black thick double-sided arrow in Fig. 2). Therefore, as a function of the insertion of the moving wedge, the CPI introduces a variable delay $\tau$ between the $\mathrm{V}$ and $\mathrm{H}$ components with very high precision and long-term stability $(\approx 1$ attosecond). We used $\alpha$-barium borate as birefringent material, with $9^{\circ}$ apex angle of the wedges and $25-\mathrm{mm}$ lateral size. This resulted in a maximum delay $\tau_{\max }=1.5 \mathrm{ps}$, corresponding to $\Delta \lambda=0.8 \mathrm{~nm}$ spectral resolution at $600 \mathrm{~nm}$. After the CPI and the sample, the light transmitted by a second polarizer $P_{2}$ (identical to $P_{1}$ but oriented at an angle $\beta$ with respect to the vertical) is detected by a photodiode connected to a data acquisition card

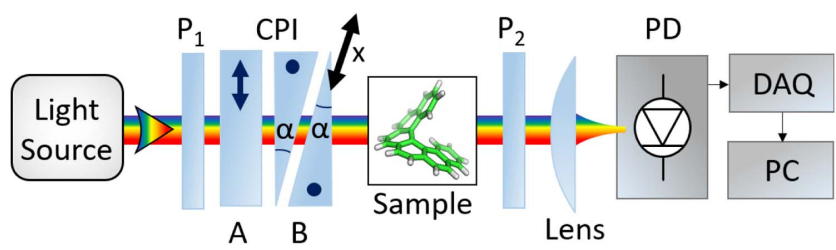

Fig. 2. Experimental setup for the time-domain SP using a CPI. $P_{1}$, $P_{2}$, linear polarizers; DAQ, data acquisition card; PC, personal computer. and a computer. Both $P_{1}$ and $P_{2}$ are mounted on a precision rotary stage for fine control (within $\approx 100 \mathrm{mdeg}$ ) of the angles. Upon scanning the CPI delay with two orientations of polarizer $P_{2}$, the chiral $\left(\beta=90^{\circ}\right)$ and calibration $\left(\beta=45^{\circ}\right)$ IGs are recorded and the $\mathrm{CB}$ and $\mathrm{CD}$ spectra are calculated according to Eqs. (6).

Both Michelson and CPI interferometers introduce a delay $\tau$ proportional to the position $x$ of the moving mirror or wedge, respectively. However, while a Michelson imparts the same delay for all the frequencies, a CPI confers a delay that is frequency dependent: $\tau(\omega)=\Delta n(\omega) \cdot \sin (\alpha) \cdot x / c$, where $\alpha$ is the wedge apex angle (see Fig. 2) and $\Delta n(\omega)$ is the frequency-dependent birefringence. In both cases, one performs an FT with respect to $x$, thus obtaining a first spectrum as a function of the spatial frequencies $f_{x}$. For a Michelson interferometer the optical frequencies $\omega$ are linearly proportional to $f_{x}$; for the CPI this relationship slightly deviates from linearity due to the aforementioned frequency dependence of the birefringence $\Delta n(\omega)$ of commonly used materials. This effect has been discussed previously [21-27]; see also Fig. 2(b) in Ref. [24]. A calibration procedure is then sufficient to retrieve the optical frequencies, using either a set of interference filters [25], a known transmission spectrum [27], or a calibrated reference spectrometer $[21,24]$.

To test the performances of our time-domain SP, we performed measurements on the two enantiomers of nickel tartrate dissolved in distilled water in a $1-\mathrm{cm}$ path-length cell at $120 \mathrm{mM}$ concentration. The green line in Fig. 3(a) shows the calibration IG $I_{\text {cal }}(x)$ for an angle $\beta=45^{\circ}$. The FT of $I_{\text {cal }}(x)$ yields $I_{\text {cal }}\left(f_{x}\right)$, which becomes the spectrum of the light transmitted by the sample $I_{\text {cal }}(\omega)$, shown as a green area in the top panel of Fig. 3(b), after calibration of the frequency axis using a set of narrowband interference filters. In Fig. 3(b) the absorption spectrum of the molecule is also reported as a black line. The chiral IGs $I_{\text {chi }}(x)$ for the $(S, S)$ and $(\mathrm{R}, \mathrm{R})$ forms of nickel tartrate, measured with $\gamma=4^{\circ}$, are shown in Fig. 3(a) as blue and red curves, respectively. One can note that: (i) the two IGs are perfectly out of phase, and (ii) they are shifted with respect to the zero optical-path difference by the same quantity (approx. 1.5 optical cycles), because, unlike $I_{\text {cal }}(\tau), I_{\text {chi }}(\tau)$ is not a symmetric function with respect to $\tau$ [see Eq. (4)]. Figure 3(b) displays, as solid (dashed) lines, the CD
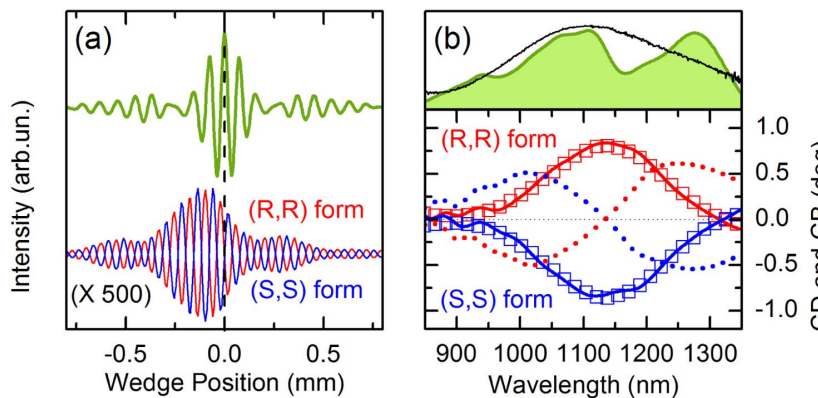

Fig. 3. (a) Calibration (green line) and chiral (blue and red lines) IGs of nickel tartrate enantiomers. Chiral IG was measured with a halogen lamp in $5 \mathrm{~s}$, averaged 10 times. (b) Top panel, excitation light spectrum transmitted by the sample and absorption spectrum of Nickel Tartrate; bottom panel, CD and CB spectra (solid and dotted lines, respectively) of ( $R, R)$ (red curves) and $(S, S)$ (blues curves) enantiomers. Red and blue squares are the $\mathrm{CD}$ spectra measured with a standard dispersive SP. 

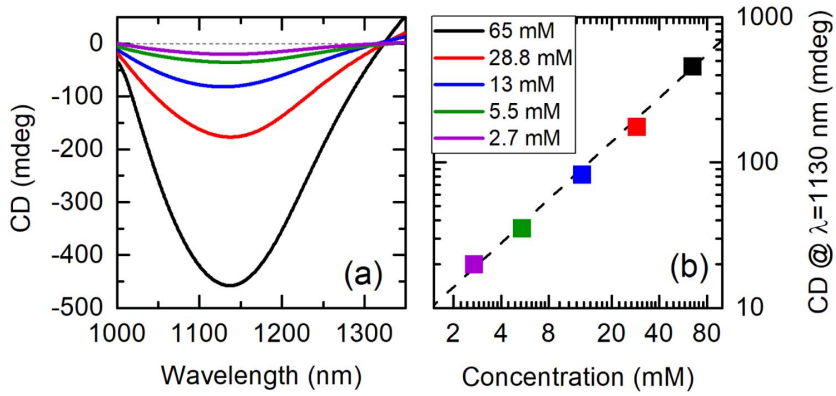

Fig. 4. (a) CD spectra for the $(S, S)$ form of nickel tartrate at different concentrations, measured with a SC laser. (b) CD signal of nickel tartrate peak at $\lambda=1130 \mathrm{~nm}$ as a function of concentration (colored squares), together with a linear fit (dotted line).

(CB) spectra of both enantiomers [(S, S) form in blue and (R, $\mathrm{R})$ form in red] obtained from the imaginary (real) part of the FTs of the chiral IGs. The CD signals measured with our timedomain SP are in excellent agreement, both in shape and in absolute value, with the signals measured by a standard dispersive SP [28], shown in Fig. 3(b) as squares.

To demonstrate the capability of our instrument to accept broadband pulses, making it suitable for ultrafast measurements of OA, we have replaced the incoherent lamp with a broadband laser. The resulting CD signals for different tartrate concentrations are plotted in Fig. 4(a), showing a good reproducibility of the shape also for low concentrations, as reflected by the constant zero crossing at $\approx 1320 \mathrm{~nm}$. Figure 4(b) shows the CD amplitude at $\lambda=1135 \mathrm{~nm}$ as a function of concentration together with a linear fit, displaying the expected scaling of the signal with concentration. These measurements illustrate the sensitivity of our time-domain SP, remarkable given the simplicity of the apparatus, but not yet at the sub-mdeg level of commercial SPs.

In this Letter, we have introduced a novel approach to the broadband measurement of $\mathrm{OA}$, combining time-domain detection with heterodyne amplification. Our method uses a birefringent common-path PDI, creating two highly stable replicas of the input light with highly pure perpendicular polarizations. The more intense replica interacting with the sample produces a CFID field, which is amplified by the other replica, acting as a phase-coherent LO. The exceptional precision of our CPI allows us to record IGs without active delay stabilization and to retrieve the $\mathrm{CD}$ and $\mathrm{CB}$ spectra using a single-pixel detector. Our device has a small footprint, is low cost, and does not require a monochromator, a PEM, or a lock-in amplifier. The method can be readily extended to the mid-IR, using $\mathrm{Hg}_{2} \mathrm{Cl}_{2}$ (calomel) as birefringent material [29].

Our time-domain approach has great potential for timeresolved OA measurements to study ultrafast stereochemical processes [30,31]. The device accepts broadband ultrashort pulses, whereas standard SP in the UV-visible allows only for monochromatic beams $[19,32,33]$. In addition, time-domain detection enables modulation of an actinic pump pulse at high repetition rates, up to the $\mathrm{MHz}$ range, as demonstrated in Refs. [25,26]. Demodulation of the signal as a function of pump-probe delay $T$ and PDI delay $\tau$ should allow extracting the pump-induced differential chiral IG $\Delta I_{\text {chi }}(\tau, T)$, from which the transient OA spectra can be obtained by FT, with much higher sensitivity than the linear measurements reported here.
Funding. H2020 European Research Council (ERC) (291198, 648615, 754802); Schweizerischer Nationalfonds zur Förderung der Wissenschaftlichen Forschung (SNF) (200020_143487).

Acknowledgment. We gratefully acknowledge fruitful discussions and experimental help from Prof. Sergio Abbate, Prof. Giovanna Longhi, Dr. Giuseppe Mazzeo, and Dr. Ettore Castiglioni.

\section{REFERENCES}

1. N. Berova, K. Nakanishi, and R. W. Woody, Circular Dichroism: Principles and Applications (Wiley, 2000).

2. L. D. Barron, Molecular Light Scattering and Optical Activity (Cambridge University, 2004).

3. N. Sreerama and R. W. Woody, Methods Enzymol. 383, 318 (2004).

4. G. D. Fasman, Circular Dichroism and the Conformational Analysis of Biomolecules (Springer, 1996).

5. J. Helbing and M. Bonmarin, J. Chem. Phys. 131, 174507 (2009).

6. H. Rhee, I. Eom, S.-H. Ahn, and M. Cho, Chem. Soc. Rev. 41, 4457 (2012).

7. P. L. Polavarapu, Principles and Applications of Polarization-Division Interferometry (Wiley, 1997).

8. N. Ragunathan, N. S. Lee, T. B. Freedman, L. A. Nafie, C. Tripp, and H. Buijs, Appl. Spectrosc. 44, 5 (1990).

9. P. L. Polavarapu, Z. Deng, and G.-C. Chen, Appl. Spectrosc. 49, 229 (1995).

10. H. Rhee, Y.-G. June, J.-S. Lee, K.-K. Lee, J.-H. Ha, Z.-H. Kim, S.-J. Jeon, and M. Cho, Nature 458, 310 (2009).

11. H. Rhee, J. Choi, and M. Cho, Acc. Chem. Res. 43, 1527 (2010).

12. L. Lepetit, G. Chériaux, and M. Joffre, J. Opt. Soc. Am. B 12, 2467 (1995).

13. H. Rhee, J. H. Ha, S.-J. Jeon, and M. Cho, J. Chem. Phys. 129, 94507 (2008).

14. I. Eom, S.-H. Ahn, H. Rhee, and M. Cho, Phys. Rev. Lett. 108, 103901 (2012).

15. K. Hiramatsu and T. Nagata, J. Chem. Phys. 143, 121102 (2015).

16. R. C. Jones, J. Opt. Soc. Am. 31, 488 (1941).

17. X. Xie and J. D. Simon, J. Opt. Soc. Am. B 7, 1673 (1990).

18. C. Niezborala and F. Hache, J. Opt. Soc. Am. B 23, 2418 (2006).

19. B. Dutta and J. Helbing, Opt. Express 23, 16449 (2015).

20. I. Eom, S.-H. Ahn, H. Rhee, and M. Cho, Opt. Express 19, 10017 (2011).

21. D. Brida, C. Manzoni, and G. Cerullo, Opt. Lett. 37, 3027 (2012).

22. F. Preda, A. Oriana, J. Réhault, L. Lombardi, A. C. Ferrari, G. Cerullo, and D. Polli, IEEE J. Sel. Top. Quantum Electron. 23, 88 (2017).

23. J. Réhault, M. Maiuri, A. Oriana, and G. Cerullo, Rev. Sci. Instrum. 85, 123107 (2014).

24. A. Perri, F. Preda, C. D'Andrea, E. Thyrhaug, G. Cerullo, D. Polli, and J. Hauer, Opt. Express 25, A483 (2017).

25. J. Réhault, F. Crisafi, V. Kumar, G. Ciardi, M. Marangoni, G. Cerullo, and D. Polli, Opt. Express 23, 25235 (2015).

26. F. Preda, V. Kumar, F. Crisafi, D. G. Figueroa del Valle, G. Cerullo, and D. Polli, Opt. Lett. 41, 2970 (2016).

27. A. Oriana, J. Réhault, F. Preda, D. Polli, and G. Cerullo, J. Opt. Soc. Am. A 33, 1415 (2016)

28. E. Castiglioni, F. Lebon, G. Longhi, and S. Abbate, Enantiomer 7, 161 (2002).

29. J. Réhault, R. Borrego-Varillas, A. Oriana, C. Manzoni, C. P. Hauri, J. Helbing, and G. Cerullo, Opt. Express 25, 4403 (2017).

30. J. Meyer-llse, D. Akimov, and B. Dietzek, Laser Photon. Rev. 7, 495 (2013).

31. J. Conyard, K. Addison, I. A. Heisler, A. Cnossen, W. R. Browne, B. L. Feringa, and S. R. Meech, Nat. Chem. 4, 547 (2012).

32. A. Trifonov, I. Buchvarov, A. Lohr, F. Würthner, and T. Fiebig, Rev. Sci. Instrum. 81, 43104 (2010).

33. L. Mangot, G. Taupier, M. Romeo, A. Boeglin, O. Cregut, and K. D. Dorkenoo, Opt. Lett. 35, 381 (2010). 